\title{
Sistema Inteligente de Apoio a Decisão no Processo de Triagem de Pacientes com Suspeita do COVID-19
}

\author{
Leonardo Ferreira Lopes ${ }^{1}$,Francisco Artannyel Pinto ${ }^{1}$, Rhyan Ximenes de Brito ${ }^{1}$ \\ ${ }^{1}$ Instituto Federal de Educação, Ciência e Tecnologia do Ceará (IFCE) \\ CE-187, s/n - Estádio, - CEP 62320-000 - Tianguá - CE - Brasil \\ \{leonnardo.fer, artannyel,rxbrito\}@gmail.com
}

\begin{abstract}
The use of Artificial Intelligence proves to be increasingly relevant, especially with regard to the accuracy of medical diagnoses. Thus, this study aimed to use intelligent agents as a tool capable of assisting in the screening of patients with suspected COVID-19, considering the initial clinical condition and the criteria established by the World Health Organization (WHO). The methodology was based on the implementation and tests with fictitious patients, considering their symptoms and the use of the textit framework JADE. Based on the results, it can be concluded that the use of intelligent agents has a lot to contribute in the search for solutions in the medical field, especially in the aid of diagnoses related to COVID-19.
\end{abstract}

Resumo. O uso da Inteligência Artificial demonstra-se cada vez mais relevante, principalmente com relação a precisão de diagnósticos médicos. Assim este estudo teve como objetivo a utilização de agentes inteligentes como ferramenta capaz de auxiliar na triagem de pacientes com suspeita de COVID-19, considerando a condição clínica inicial e os critérios estabelecidos pela Organização Mundial da Saúde (OMS). A metodologia foi baseada na implementação e testes com pacientes fictícios, tendo em vista os sintomas dos mesmos e na utilização do framework JADE. Com base nos resultados pode-se concluir que o uso de agentes inteligentes tem muito a contribuir na busca por soluções na área médica em especial no auxílio de diagnósticos relacionados a COVID-19.

\section{Introdução}

O novo coronavírus provoca uma doença respiratória com sintomas similares ao da gripe ou mesmo de um resfriado comum, deste modo, há uma dificuldade no diagnóstico preciso e definitivo da doença causada pelo vírus e conhecida como COVID-19. O vírus não é seletivo, podendo infectar desde crianças à idosos, colocando em risco a vida principalmente dos mais velhos e portadores de alguma comorbidade.

A triagem dos pacientes realizada por médicos é feita após proposições hipotéticas de diagnósticos, usando como base os dados recolhidos sobre os problemas de saúde do mesmo, que são analisados e comprovados através de exames mais minuciosos [Lobo 2017]. Em uma avaliação de uma pessoa que apresente febre, tosse, dores no corpo um diagnóstico de gripe pode ser comum. Porém com um posterior agravamento do quadro clínico, surge a necessidade de exames mais detalhados para comprovações.

Este trabalho tem como objetivo principal auxiliar profissionais da saúde no processo de triagem de pacientes com suspeita de COVID-19, através da elaboração de um 
sistema com ênfase em agentes inteligentes baseado em perfis de sintomas que distinguem a COVID-19 de doenças respiratórias através do grau dos sintomas relacionados.

Este trabalho está organizado da seguinte forma: a Seção 2 apresenta os trabalhos relacionados, na Seção 3 é apresentada a fundamentação teórica. Em seguida na Seção 4 apresenta-se a metodologia. Na Seção 5 os resultados e discussões e finalmente, conclusões e trabalhos futuros na Seção 6.

\section{Trabalhos Relacionados}

Esta seção apresenta uma revisão bibliográfica com trabalhos relacionados a utilização da Inteligência Artificial como instrumento auxiliador no pré-diagnóstico médico de vários problemas em saúde.

[de Brito et al. 2019] realizou-se um estudo através da implementação da rede MLP (Multilayer Perceptron), objetivando usá-la como auxílio na identificaçãoo de pessoas com ou sem problemas cardíacos, com ênfase no treinamento e teste para classificação desses indivíduos. A metodologia foi implementada com base em 270 amostras e 14 atributos. Foram feitos 10 treinamentos com os dados balanceados e normalizados. Os resultados foram analisados estatísticamente com base nos percentuais de acertos e erros da rede implementada, obtendo-se uma medida da qualidade atingida.

[da Silva et al. 2017] desenvolveram um trabalho com o objetivo de definir um sistema fuzzy para auxiliar na análise dos riscos de uma mulher desenvolver ou já ser portadora do câncer de mama, tendo como medida de avaliação três variáveis que são: a idade, os sintomas e os fatores de risco.

[Kermany et al. 2018] elaboraram uma ferramenta de diagnóstico com base em uma estrutura de aprendizado profundo para a triagem de pacientes com doenças retinianas ofuscantes tratáveis comuns. A estrutura utiliza o aprendizado por transferência, que treina uma rede neural com uma fração dos dados das abordagens convencionais. Aplicando essa abordagem a um conjunto de dados de imagens de tomografia de coerência óptica, demonstrou-se desempenho comparável ao de especialistas humanos na classificação da degeneração macular relacionada à idade e edema macular diabético.

[Rocha et al. 2020] propos um modelo de previsão de curto prazo baseado em Análise dos Componentes Principais (PCA) e Redes Neurais Artificiais (RNAs) com a capacidade de estimar o número de casos e de óbitos causados pelo SARS-CoV-2. O modelo é baseado em dados, onde toda inferência é feita a partir do conhecimento descoberto. O estudo apresentou resultados para o estado do Pará e Brasil, com séries temporais que serviram de base para analisar o impacto na capacidade de atendimento nos leitos de Unidade de Terapia Intensiva (UTI), servindo de suporte na tomada de decisão por parte dos órgãos de vigilância em saúde.

[de Almeida et al. 2017] desenvolvou uma ferramenta de software denominada SIMUNOS, cujo objetivo foi simular o comportamento do sistema imunológico humano utilizando agentes inteligentes. A fim de avaliar a proposta foram feitos experimentos usando como métricas a variação na população de antígenos e neutrófilos. Os resultados demonstram a viabilidade do uso de Modelagem e Simulação Baseada em Agentes para estudar sistemas complexos.

[Ximenes et al. 2019] propôs que os Sistemas Nebulosos são adequados para a 
manipulação de informações subjetivas, como no cenário exposto na sua pesquisa. Teve como principal objetivo definir um sistema fuzzy para auxiliar no diagnóstico da menigite, tendo como medida de avaliação os sintomas característicos da doença abordada.

[de Brito et al. 2019] realizou um estudo com Redes Neurais Artificiais através da implementação da rede MLP (Multilayer Perceptron), objetivando usá-la como auxílio na identificação de pessoas com ou sem problemas cardíacos, com ênfase no treinamento e teste para classificação desses indivíduos. A metodologia foi implementada com base em 270 amostras e 14 atributos, utilizando a ferramenta Matlab R2015a. Os resultados foram analisados estatisticamente com base nos percentuais de acertos e erros da rede implementada, obtendo-se uma medida da qualidade atingida.

\section{Fundamentação Teórica}

Esta seção apresenta a fundamentação teórica relacionada a Agentes Inteligentes e a COVID-19.

\subsection{Agentes Inteligentes}

A Inteligência Artificial (IA) é um ramo da Ciência da Computação que se propõe a desenvolver sistemas que simulem a capacidade humana de percepção de um problema, identificando seus componentes para com isso, resolver problemas com base em decisões [Lobo 2017]. Nesse sentido sabe-se que um agente inteligente é um sistema computacional de software, hardware ou misto, atua com base em suas percepções, tendo ações autônomas, para chegar ao objetivo desejado.

Segundo [Russel et al. 2013] basicamente existem 5 tipos de agentes inteligentes, cada um com seu funcionamento interno, são eles:

- Agente Reativo Simples: selecionam ações com base somente na percepção atual, $\mathrm{O}$ funcionamento do agente reativo é baseado em regras de condição-ação: se (condição) então ação. O agente funciona apenas se o ambiente for completamente observável e a decisão correta puder ser tomada com base apenas na percepção atual.

- Agente Reativo Baseado em Modelo: é o modo mais efetivo de lidar com a possibilidade de observação parcial, assim o agente monitora a parte do mundo que ele não pode ver agora. Assim o agente deve manter algum tipo de estado interno que dependa do histórico de percepções e assim reflita pelo menos alguns dos aspectos não observados do estado atual.

- Agente Baseado em Objetivo: conhecer algo sobre o estado atual do ambiente nem sempre é suficiente para decidir o que fazer. A decisão correta depende de onde o táxi está tentando chegar. Em outras palavras, da mesma forma que o agente precisa de uma descrição do estado atual, também precisará de alguma espécie de informação sobre objetivos que descreva situações desejáveis - por exemplo, estar no destino do passageiro.

- Agente Baseado em Utilidade: sozinhos, os objetivos não são realmente suficientes para gerar um comportamento de alta qualidade na maioria dos ambientes, por exemplo, existem muitas sequências de ações que levarão o táxi até seu destino, mas algumas são mais rápidas, mais seguras, mais confiáveis ou mais econômicas que outras. Os objetivos simplesmente permitirão uma distinção binária crua entre 
estados "felizes" e "infelizes", enquanto uma medida de desempenho mais geral deve permitir uma comparação entre diferentes estados do mundo, de acordo com o grau exato de felicidade que proporcionariam ao agente.

- Agentes com Aprendizagem: um agente de aprendizado pode ser dividido em componentes conceituais. Assim a distinção mais importante dar-se entre o elemento de aprendizado, responsável pela execução de aperfeiçoamentos e o elemento de desempenho, responsável pela seleção de ações externas. O elemento de desempenho recebe percepções e decide sobre ações. O elemento de aprendizado utiliza realimentação do componente crítico sobre como o agente está funcionando e determina de que maneira o elemento de desempenho deve ser modificado para funcionar melhor no futuro.

\subsection{COVID-19}

A COVID-19 é uma doença provocada por um vírus da família Coronavírus, SARS-Cov-2 identificada pela primeira vez em Wuhan, na China em dezembro de 2019, de acordo com a Organização Mundial da Saúde (OMS). A COVID-19 é uma doença respiratória tendo o sistema respiratório largamente atacado pelo vírus ocasionando dificuldades respiratórias podendo levar a morte [MS 2020]. O vírus tem um formato de coroa, daí vem o nome coronavírus.

Entre os vírus que compõem essa família e que causam a COVID-19 encontra-se o que causa a MERS, a síndrome respiratória do Oriente Médio sendo uma doença respiratória aguda grave causada pelo coronavírus MERS-CoV, sendo identificada pela primeira vez em setembro de 2012 na Arábia Saudita. E o que causa a SARS, Síndrome Respiratória Aguda Grave doença respiratória aguda grave causada pelo coronavírus SARS$\mathrm{CoV}$ semelhante a gripe causada pelo vírus Influenza. Os primeiros casos ocorram na China em 2002 na província de Guangdong [Brenda 2020].

Os sintomas relatados por pacientes e apontados pela OMS e Ministério da Saúde (MS) são: tosse, febre, dor de garganta e dificuldade respiratórias, sintomas esses semelhantes a outras doenças respiratórias causadas por outros agentes como a gripe comum provocado pelo vírus Influenza e o resfriado comum que pode ser provocado por diversos vírus como adenovírus, desde modo dificultando um diagnóstico preciso baseado apenas nos sintomas [MS 2020]. A Tabela 1 expõe os principais sintomas do coronavírus, gripe e resfriado.

\section{Metodologia}

A abordagem realizada considerou simulações com pacientes fictícios e o desenvolvimento do sistema ocorreu em três etapas: na etapa 1 definiu-se o projeto e a criação do Performance, Environment, Actuators, Sensors (PEAS), ou seja, desempenho, ambiente, atuadores e sensores; na etapa 2 implementou-se o sistema usando o framework JADE e por fim à etapa 3 realizou-se os testes e as simulações.

\subsection{Definição do projeto e criação do PEAS}

Nessa fase foi definido o projeto ressaltando a importância de instrumentos computacionais no combate a COVID-19, assim como o PEAS, conforme Tabela 2, com a finalidade de especificar o ambiente de atuação, performance, atuadores e sensores do agente, almejando padrões de qualidade e de sucesso. 
Tabela 1. Sintomas Coronavírus, Gripe, Resfriado

\begin{tabular}{cccc}
\hline Sintomas & Coronavírus & Gripe & Resfriado \\
\hline Febre & Comum & Comum & Raro \\
Cansaço & Às vezes & Comum & Às vezes \\
Tosse seca & comum & Comum & Leve \\
Espirros & Raro & Raro & Comum \\
Dores no corpo e mal estar & Às vezes & Comum & Comum \\
Coriza e nariz entupido & Raro & Às vezes & Comum \\
Dor de garganta & Às vezes & Às vezes & Comum \\
Diarreia & Raro & Às vezes (crianças) & Raro \\
Dor de cabeça & Às vezes & Comum & Raro \\
Falta de ar & Às vezes & Raro & Raro \\
\hline
\end{tabular}

Tabela 2. PEAS - Sistema de Triagem COVID-19

\begin{tabular}{cc}
\hline Perfomance & Distinção da COVID-19 de outras doenças respiratórias \\
Environment & Consultório, hospital, posto de saúde \\
Actuators & Uso de telas para exibir pré-diagnósticos \\
Sensors & Inserção de sintomas através do teclado \\
\hline
\end{tabular}

Vale ressaltar que para este trabalho foi considerado o agente reativo simples pelo fato do mesmo não considerar o histórico de percepções, ou seja, ele seleciona a ação a partir da percepção atual do ambiente [de Sousa Ximenes et al. 2017].

\subsection{Implementação do sistema usando o framework JADE}

O desenvolvimento do sistema baseou-se na utilização de agentes inteligentes. A escolha do tipo agente considerou inicialmente que o mesmo fosse capaz de tomar uma decisão com base nos conhecimentos no momento, logo, optou-se pelo agente reativo simples, implementado em linguagem Java através do Java Agent Development Framework (JADE).

JADE é um framework para construção de sistemas multiagentes. Os agentes são implementados através de classes que gerenciam os comportamento do mesmo. Existem outros frameworks para o desenvolvimento de agentes como no caso do Python Agent Development framework (PADE) para implementações em linguagem Python.

Salienta-se que o sistema construído é composto por um único agente nomeado como DoctorAgent, responsável por perceber os sintomas listados na Tabela 1 baseados na entrada feita por uma profissional de saúde.

A Tabela 3 apresenta os critérios utilizados como base pelo agente DoctorAgent que irá avaliar o paciente, atribuindo um pré-diagnóstico "suspeita de COVID-19 é alta", caso as condições clínicas se enquadrem nos parâmetros estabelecidos pela MS, senão, o seguinte pré-diagnóstico "suspeita de COVID-19 é baixa".

Todos os casos seguirão a mensagem "para um pré-diagnóstico preciso, exames clínicos se mostram necessários", visto que os critérios utilizados pelo agente inteligente 
Tabela 3. Critérios Utilizados para Triagem

\begin{tabular}{|c|c|c|c|c|}
\hline & & Características Clínicas & & Risco Epidemiológico \\
\hline \multirow{3}{*}{ Febre } & $\mathrm{OU}$ & $\begin{array}{l}\text { pelo menos um sinal/sintoma } \\
\text { respiratório (tosse, dificul- } \\
\text { dade para respirar, batimento } \\
\text { de asa de nariz, entre outros). }\end{array}$ & $\mathrm{E}$ & $\begin{array}{l}\text { Histórico de contato próximo do } \\
\text { novo coronavírus em laboratório, } \\
\text { nos últimos } 14 \text { dias antes do início } \\
\text { dos sinais ou sintomas }\end{array}$ \\
\hline & $\mathrm{E}$ & $\begin{array}{l}\text { pelo menos um sinal/sintoma } \\
\text { respiratório (tosse, dificul- } \\
\text { dade para respirar, batimento } \\
\text { de asa de nariz, entre outros). }\end{array}$ & $\mathrm{E}$ & $\begin{array}{l}\text { Histórico de contato próximo de } \\
\text { caso suspeito para o novo coro- } \\
\text { navírus (COVID-19), nos últimos } \\
14 \text { dias antes do início dos sintomas }\end{array}$ \\
\hline & $\mathrm{E}$ & $\begin{array}{l}\text { pelo menos um sinal/sintoma } \\
\text { respiratório (tosse, dificul- } \\
\text { dade para respirar, batimento } \\
\text { de asa de nariz, entre outros) }\end{array}$ & $\mathrm{E}$ & $\begin{array}{l}\text { Histórico de viagem para área com } \\
\text { transmissão local, de acordo com a } \\
\text { OMS nos últimos } 14 \text { dias antes do } \\
\text { início dos sinais ou sintomas. }\end{array}$ \\
\hline
\end{tabular}

não conseguem fechar um diagnóstico tão preciso quanto uma análise laboratorial, logo o agente ainda tem uma margem maior de erros do que exames mais detalhados. Porém auxiliará profissionais de saúde na tomada de decisões.

\subsection{Testes e Simulações}

Para a realização dos testes utilizou-se o seguinte procedimento: com a ferramenta inicializada o usuário fornece ao agente DoctorAgent dados sobre os sintomas apresentados pelos pacientes fictícios, bem como os riscos, assim os dados são recebidos com valores 0 ou 1 , negativo ou positivo como resposta.

O agente irá traçar um perfil clínico e o julgará segundo critérios estabelecidos pela MS referenciados na Tabela 3, em seguida exibirá o resultado. Foram realizadas 15 simulações com pacientes fictícios atribuindo-se diferentes quadros clínicos aos mesmos.

\section{Resultados e Discussões}

Nesta seção foram destacados 3 dos resultados gerados pelo agente DoctorAgent obtidos através das simulações realizadas com vários pacientes fictícios.

Assim para o paciente fictício $\mathrm{X}$ de 35 anos observou-se os seguintes sintomas com base na percepção do agente DoctorAgent, relacionados com os critérios de triagem: febre, cansaço, dor no corpo e teve contato com alguém suspeito de COVID-19. Dessa forma de acordo com a Figura 1, foi diagnósticado que o paciente tem uma alta probabilidade de estar com a COVID-19, obtidos com base nos critérios da triagem. Com essa suspeita um profissional de saúde pode encaminhá-lo para um exame laboratorial para confirmação ou descarte da suspeita.

Para o paciente fictício Y de 24 anos, foram simulados os seguintes sintomas: tosse seca, espirros, dor no corpo, coriza, febre, o paciente não viajou para nenhuma área com casos de COVID-19, nem teve contato com pessoas com diagnóstico confirmado de COVID-19. Assim o agente DoctorAgent diagnósticou que a probabilidade do paciente está com COVID-19 é baixa, conforme a Figura 2. Dessa forma cabendo ao profissional de saúde decidir o dignóstico e que tratamento prescrever ao paciente. 


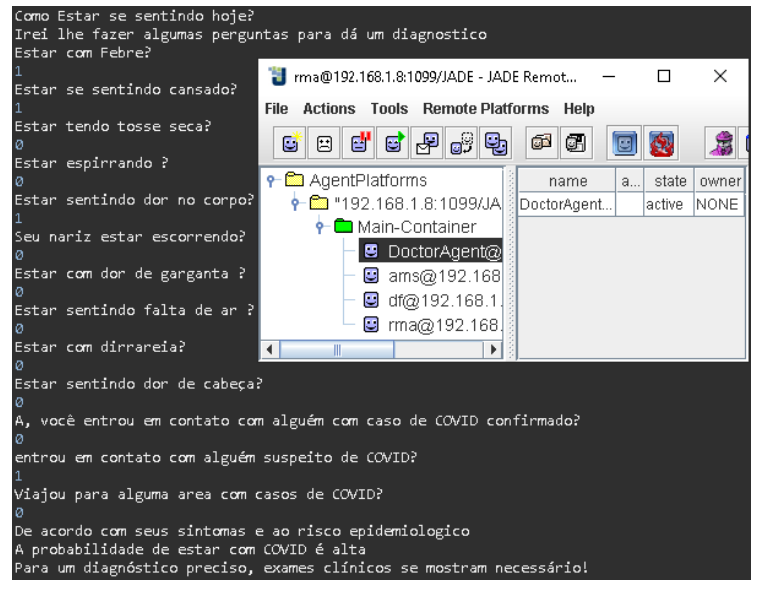

Figura 1. Paciente fictício $X$

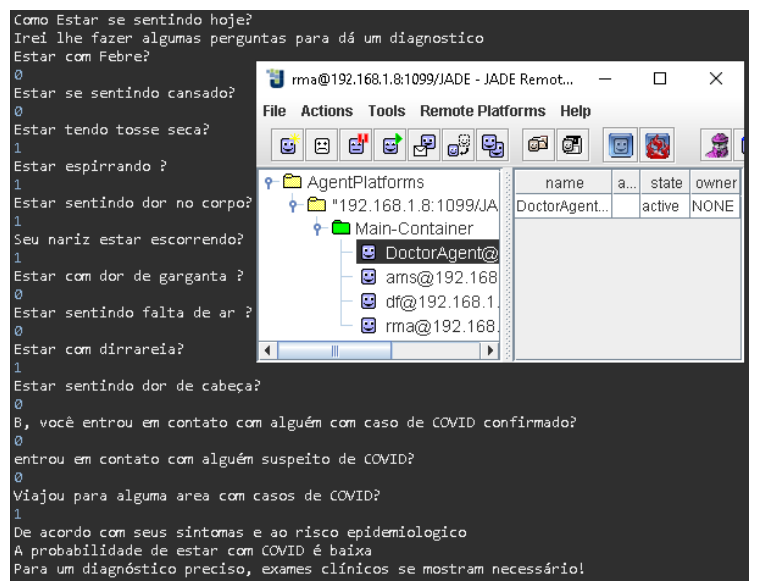

Figura 2. Paciente fictício $Y$

Já para o paciente fictício $\mathrm{Z}$ de 78 anos que apresentou os seguintes sintomas: febre, espirros, tosse, coriza e dificuldade de respirar. O agente DoctorAgent conforme observado na Figura 3 pré-diagnosticou que o paciente tem baixa probabilidade de está com COVID-19.

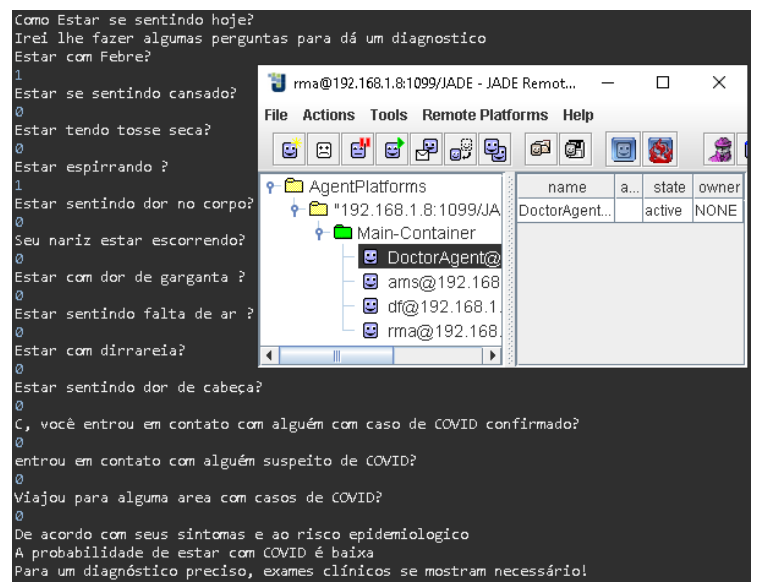

Figura 3. Paciente fictício Z 


\section{Conclusões e Trabalhos Futuros}

O uso de agentes inteligentes configura-se como um importante instrumento para auxiliar profissionais da saúde no diagnóstico de doenças, principalmente para encaminhamentos à exames complementares ou até mesmo para prescrição de tratamentos medicamentosos. Nesse sentido observou-se que o agente teve um bom desempenho como uma ferramenta de pré-diagnóstico.

Como trabalho futuro sugere-se o aperfeiçoamento do agente com testes baseados em dados reais com acompanhamento de um profissional da saúde de forma a aprimorar sua capacidade de diagnóstico.

\section{Referências}

Brenda, L. T. (2020). Coronavírus e síndromes respiratórias agudas. [Online; acessado em: 23-Maio-2020].

da Silva, P., de Brito, R., de Sousa Ximenes, J., and de Sousa, R. (2017). A aplicaçao da lógica de fuzzy no auxilio do diagnóstico do câncer de mama.

de Almeida, M., de Freitas, P., Barros, J., Ribeiro, G., da Silva, M., and Matos, R. (2017). Uma ferramenta baseada em agentes inteligentes aplicada ao estudo do sistema imunológico humano. In Anais do IV Encontro Nacional de Computação dos Institutos Federais, pages 21-24, Porto Alegre, RS, Brasil. SBC.

de Brito, R. X., de Sousa Ximenes, J. N., da Silva, P. H. A., and de Sousa, R. N. (2019). Sistema de análise de dados através de uma rede neural artificial mlp na prediç ao de doença cardiaca. ANAIS ELETRÔNICOS CAIS TECH 2019, page 102.

de Sousa Ximenes, J. N., de Lima Campos, G. A., and de Vasconcelos Silveira, F. R. (2017). Promon: agente de diagnóstico e monitoramento de falhas para agentes racionais. Revista Brasileira de Computação Aplicada, 9(1):84-96.

Kermany, D. S., Goldbaum, M., Cai, W., Valentim, C. C., Liang, H., Baxter, S. L., McKeown, A., Yang, G., Wu, X., Yan, F., et al. (2018). Identifying medical diagnoses and treatable diseases by image-based deep learning. Cell, 172(5):1122-1131.

Lobo, L. C. (2017). Inteligência artificial e medicina. Revista Brasileira de Educação Médica, 41(2):185-193.

MS, M. S. (2020). Sobre coronavirus. [Online; acessado em: 24-Maio-2020].

Rocha, J. E. C. d., SOUZA JÚNIOR, G. N. d., Brito, S. R. d., Folador, A. R. C., Ramos, R. T. J., Braga, M. d. B., Botelho, M. d. N., et al. (2020). Redes neurais artificiais na previsão de contágio e óbitos por covid-19: um estudo no estado do pará, brasil.

Russel, S., Norvig, P., et al. (2013). Artificial intelligence: a modern approach. Pearson Education Limited.

Ximenes, J., de Sousa, R., and de Brito, R. (2019). Sistema fuzzy como instrumento de auxílio no pré-diagnóstico da meningite. In Anais da VII Escola Regional de Computação Aplicada à Saúde, pages 264-269, Porto Alegre, RS, Brasil. SBC. 